\title{
Morphological reasoning of paraaortal surgical access for an abdominal aortic neural plexus in pet dogs
}

\author{
Oleg Romanovich Skubko ${ }^{1, *}$ and Olga Nikolaevna Shushakova ${ }^{2}$ \\ ${ }^{1}$ Chair of Diagnosis, Internal Non-Communicable Diseases, Pharmacology, Surgery and Obstetrics, Omsk State Agrarian University \\ named after P.A. Stolypin, Omsk, 644122, Russia \\ ${ }^{2}$ Chair of Anatomy, Histology, Physiology and Pathology, Omsk State Agrarian University named after P.A. Stolypin, Omsk, 644122 , \\ Russia
}

\begin{abstract}
Despite a great number of studied and development of new treatment of various diseases, there is a lack of effective surgical access pathways to an abdominal autonomic nerve in small predatory animals. The study is purposed to develop a morphologically reasonable, species-specific surgical access of paraaortic injection of local anesthetics into an area of localization of abdominal autonomic nerves in pet dogs. The subjects of the study were corpses of mature mesomorphic pet dogs fallen of non-communicable diseases. The complex of morphological methods used in this research is as follows: normal and subtle dissection on the academic Vorobyov's method, classic methods of production of light-optical histological medications, morphometry. With the aim of developing injection access to abdominal autonomic nerves, an injection of colored latex solution with its further dissection was used. In our investigations skeletonangiotopical relations, macro-microanatomical and histological constitution of abdominal nerves of aortal plexus were studied. Epineural structures was in pet dogs were characterized. The results of macromicroanatomical and histological studies allowed to develop a technique of paraaortal surgical access to an abdominal aortal nervous plexus in pet dogs. The proposed technique of paraaortal surgical access for a block of an abdominal aortal nervous plexus allows for full coverage an area where the sources of abdominal organs innervation are located.
\end{abstract}

\section{Introduction}

To relieve pain during surgical operations in small predatory animals, most authors recommend to use an anesthesia and a neuroleptanalgesia. Rarely local anesthesia is preferred. Mostly to relieve pain in abdominal and pelvic inner organs, peridural or subarachnoidal surgical accesses for spinal anesthesia conduction and massive intravascular injection of novocaine and lidocaine are suggested [1-6].

In the field of surgical access, development of pathogenetic block of autonomous innervation centers and reviewing their impact on a dog's organism belongs to Russian scientists [7, 8].

The way forward in this field of veterinary were investigations by L.G.Smirnov [9] and V.V.Reshetnyak [10]. Their experimental and clinical studies allowed for development of an intraperitoneal way of Novocain injection in dogs for intraperitoneal procaine block conduction after experimental laparotomy and intestinal loop eventration.

However, the suggested ways of sagittal jet irrigation of mesentry and mesovarion with local anesthesia liquids did not achieve desired anesthetic and pathogenic effect and were unsafe due to a risk of a drug-induced intoxication because of demanded injection of a large quantity of anesthetic drug in an intensive tissue and vascular resorption area. For this reason, they were not widespread in clinical practice.

\section{Research objective}

The research objective was to develop a species-specific, morphofunctionally justified surgical access for conduction of paraaortal injection of local anesthetic solutions in the area of into an area of localization of abdominal autonomic nerves in pet dogs.

\section{Materials and methods}

With the aim of investigation of anatomic-topographical specificities of pelvic nerves and development of the surgical access technique for intersurgical block, we used methods as follows: macro-microdissection on V.P.Vorobyov and posthumous injection of latex solution colored with black ink into circumvascular loose fibrous connective tissue of a ventral aorta. For histostructural investigations tinction of histologic section with hematoxylin and eosin on Mallory, Veigert, Van-Girzon, Nisl and impregnation on Bilshovsky-Gross were used [11].

* Corresponding author: or.skubko@omgau.org 
A total number of morphological preparations produced was 190. Corpses of dogs mixed with unknown breeds of mesomorphic type fallen of the reasons not connected with diseases and injuries of abdominal and pelvic areas served as material for the research.

\section{Results}

It has been established that the sources of formation of abdominal aortal plexus (preganglionic branches of a frontier sympathetic trunk organized in a big and a small celiac, lateral and ventral intermesenteric and lumbar splanchnic nerves) are located in close proximity to each other in an osteotopical projection of the 12-th - the 2-nd lumber vertebra and is incarcerated here in circumvascular loose fibrous connective tissue in the initial section of abdominal aorta.

Abdominal sections of celiac nerves are located in close proximity to a frontier sympathetic trunk close to a dorsolateral wall of an abdominal aorta at the level of the 1-st - 4-th lumber vertebra. Cephalic and caudal lumbar splanchnic nerves achieve a dorsal surface of an abdominal aorta in an osteotopical projection of the 2-nd - 4-th lumber vertebra. Intermesenteric nerves pass along on lateral and ventral surfaces of an abdominal aorta. In relation to the vertebral column they are located at the level of the 1-st - 5-th lumber vertebra.

Having multiple interlinkages, intermesenteric nerves form a complicated intermesenteric plexus, which alongside with big and small celiac, cephalic and caudal lumbar splanchnic nerves, while innervating organs of GI tract, participates in construction of nephritic and testicular (ovarian in females) neuroplexes, which form in an initial section of cognominal blood vessels. Ventral muscles of a vertebral column in this area have small volume; interfascial compartments are open and filled with loose fibrous connective tissue. Paraneural loose fibrous connective tissue in the area of the lateral surface of lumbar vertebrae contains large amount of yellow fibers (relative volume is up to $1.2 \%$ ).

In a circumvascular area of a plexus's localization, the relative volume of yellow fibers ranges from $0.4 \%$ to $1.5 \%$ in various parts. This particularity determines injected solutions diffuse freely over long distances along aorta and ventral surface of vertebrae of lumbar spine column. It also lets this tissue to repair the spatial organization after stretching.

Multilayer spatial organization of an abdominal aortal plexus is provided with a connective-tissue carcass, basic substance of which contains large amount of cross-cutting collagen fibers (to 16,3\%) and includes much white adipose tissue, which represents to $3 / 4$ of volume of a connective-tissue layer.

An epineurial connective-tissue capsule, which mostly consists of collagen fibers, is modestly developed in all nerves under research. Its width is to $130 \pm 26.4$ $\mathrm{mcm}$ at the level of ventrolateral surface of vertebrae and to $38.5 \pm 7.4 \mathrm{mcm}$ near the dorsal surface of abdominal aorta. Epineurium of nerves located at lateral surfaces of abdominal aorta has the minimal thickness $-27.6 \pm 4.2$ $\mathrm{mcm}$.

Ganglion of a plexus are surrounded with a capsule, thickness of which is from $93.4 \pm 11.5 \mathrm{mcm}$ to $273.7 \pm 16.3$ $\mathrm{mcm}$. This capsule consists mostly of collagen fibers, which are connected with fibers of surrounding loose fibrous connective tissue. A large amount of lipocytes was also found around them. Development of a connective-tissue capsule around the main elements of an abdominal aortal plexus alongside with their isolation with adipose tissue demands during anesthesia to inject solution directly into the plexus as close to nerve guides as possible.

Taking into account anatomic-topographical and histostructural features of neural and paraneural structures of an abdominal aortal plexus, the technique of paraaortal surgical access for conduction of its block is suggested, which is applicable after abdominal incisions during invasive diagnostic abdominal and pelvic operations. Indications for its use are as follows: impossibility of transsomatic accesses (occurrence of purulonecrotic locus and other destructive changes of tissues in lumbar region), absence of primary indications for a block, which becomes obvious during following surgical interference, and necessity of the most accurate manual and visual control of the position of a needle, its direction and the depth of its insert during a manipulation.

Interoperational paraaortal block of an abdominal aortal plexus involves introducing the local anesthetics solutions either before or after implementation of operation modalities (depending on a clinical situation, strategy and tactic of treatment). The technique of paraaortal surgical access for conduction of a block is as follows: mesentery and all connected gastrointestinal tract organs are laterally shifted to $2-3 \mathrm{~cm}$ (to the left or to the right) with blades imposed through an incision in an abdominal wall into a cavity or with fingers isolated with a surgical glove. In this way, the point at which an abdominal parietal layer shifts to a visceral exposes itself at a dorsal abdominal wall in the area of a mesenteric root.

The angle of injection is 15-25 degrees to an internal surface of a dorsal abdominal wall. The needle is slowly moved towards loose fibrous connective tissue along an abdominal aorta in cephalic and then in caudal direction. An adaptor of a PVC tube is connected to a syringe and 3-7 $\mathrm{ml}$ of solution is injected in each direction. The sequence of directions depends on clinical need. In cases of localization of pathological loci in the area of cephalic sections of gastrointestinal tract and large digestive glands, when dominated effect of a block to a celiac and mesentericus cranialis is recommended, paraaortal access is conducted with injection of a needle and anesthetic solution in cephalic direction.

If a pathological locus is in caudal section of a gastrointestinal tract or in genitourinary organs (except kidneys and ovaries), paraaortal access for conduction of a block of an inferior mesenteric plexus hypogastrion nerves is conducted with injection of a needle and solutions in caudal direction. If a pathological locus is in the area of a small bowel, ileocecal area, cephalic section 
of a segmented intestine, ovaries and kidneys, paraaortal access is conducted with injection of a needle and solutions of local anesthetics into an area of a "root" of mesocolon aiming to block a intermesenteric, ovarian and nephritic plexuses.

When using the suggested technology in experiment, colored latex solution coagulated around gangliated cords and their branches that go from caudal thoracal and lumbar sympathetic ganglion to intermesenteric nerves and their connections at the level of caudal edge of the $11^{\text {th }}$ thoratic vertebra to cephalic edge of the fifth thoratic vertebra.

In the projection of the same sections, solution spreads vertically in paravertebral and periaortic areas, infiltrating loose fibrous connective tissue at the nerves of celiac, mesentericus cranialis, intermesenteric and inferior mesenteric plexuses linking to hypogastric nerves. There is no necessity of injection to the right and to the left of the medial longitudinal axis (and ventral aorta) as when being injected from the one side color liquid spread all over the net of abdominal aortic plexus.

\section{Discussion}

Paranephric block, developed by I.Y. Tikchonin in 1951 for horses and later applied to other animal species [9] is not targeted and suggests injection of novocaine solution into a perinephric adipose capsule which has inconstant edges in different animal spices as well as in different stages of life of a concrete animal. With that an inexact surgical access is suggested, which is weakly oriented in depth and direction $(5-10 \mathrm{~cm}$ for hoofed mammals and $1,5-3 \mathrm{~cm}$ for all canids) and requires a long period of individual training of every surgeon for development of indescribable personal experience of orientation in relevant tissues of different spices and even breeds of animals. For large animals with conspicuous paranephric fat, a mistake within 5-7 $\mathrm{mm}$ in depth and the place of injection, which is quite possible when using this technique, doesn't play any significant role.

However, for small breeds of dogs it can lead at the best - to a "downfall" of anesthetic solution into an abdominal cavity and ineffectiveness of the manipulation and at the worst - to traumas of abdominal organs and unjustified intoxication of the whole organism. Besides, the area of distribution of solution, injected using suggested methods, covers mainly renal plexus and only partially reaches other nervous components of an abdominal aortic plexus. Listed limitations obviously reveal that surgical access used for block by I.Y. Tikchonin cannot be absolutely recommended for block of abdominal aortic plexus in domestic dogs.

Developed by B.A. Bashkirov, translumbar block of lumbar inner nerves and a number of other nerve guides of this area was originally supposed to be used only for large-sized hoofed animals. Later this technique became used for carnivores as well [4, 7, 8]. However, a tangential injection towards a body of vertebra is doable only in large-sized animals, whose lateral planes of bodies of lumbar vertebrae are of significant size and a thoracolumbar section of a vertebral column is relatively inactive (hoofs, pigs, some "giant" breeds of dogs).

Small carnivores (fur bearers, cats, small and middle breeds of dogs) are significantly mobile and have smallsized elements of a thoracolumbar section of a skeleton. Besides, correlation between width of a body of vertebra and width of soft tissues of a spine which a needle should go through before touching it in dogs and cats is equal to $1: 1,9-2,1$, in distinction from this in most of artiodactyles, which is in this case equal to $1: 1,2-1,3$ with insignificant mobility of a lumbar section of a corpus $[5,7,8,12]$.

It is hugely complex to inject a needle tangentially in exactly a body of a vertebra through such a layer of permanently large-amplitude moving tissue. Medially located towards a muscle layer intertransverse ligaments of lumbar vertebrae, which have a structure of thick fibrous formed connective tissue close in its penetrance to aponeuroses, it can also become a barrier for extension of solutions injected through dorsal muscles.

Abovementioned morphofunctional particularities of small carnivores complicate an objective monitoring of delivery of anesthetic solutions directly to the sources of autonomous innervation of ovaries and uterus, create conditions of most extension of solutions either in spinal muscles of a lumbar section of a corpus or in an abdominal plexus. Moreover, there is a risk to injure an aorta and cavernous organs because of possibility to lose depth and direction control of an injection.

The suggested technique of paraaortal surgical access is much more accurate ant safe of those early used. Due to targeting of an injection, it is possible to use less quantity of anesthetic however covering topographically exact and anatomically confirmed area of localization of the sources of autonomous innervation of abdominal and pelvic organs. There are no complex insular fascial boxes in this area, preperitoneal cribriform tissue is always developed and epineurium is significantly thinner than in nerves of close areas.

Enumerated technical methods of access and morphofunctional patterns of the areas of their usage determine free diffusion of solution alongside ventral surface of bodies of lumbar vertebrae, an abdominal aorta with paravertebral plexuses of autonomous nerves allocated around. This is especially important in "wide" theranostic laparatomies, prolonged and complicated abdominal and pelvic operations. Minimization of an injection trauma and exclusion of a risk of organ injuries became possible due to absolute control of injection.

The method of lumbar block of solar plexus in dogs and cats suggested by O.R. Skubko and coauthors [7] provides for spred of injected solution only in the localization area of large and small splanchnic nerves, components of a solar plexus and cephalic sections of intermesentericnerves.

The drawback is farness of influenced autonomous centers from caudal sections of gastrointestinal tract and urogenital organs. This decreases effectiveness of the O.R. Skubko's lumbar block during treatment manipulations with colon, ovaries and uterus as therapeutic effect is more significant when those parts of a nervous system, which are topographically nearest to a 
pathological locus and innervate it are effected by Novocain block [1, 2, 5-10].

This drawback is fully dismissed in the paraaortal access now proposed for a domestic dog. It is significant by targeted delivery of medication solutions to the area of localization of ganglion and plexuses, which are morpho-functionally responsible for innervation of all gastrointestinal tract, ovaries and uterus and are anathomotopographically close to them.

Surgical access of intra-abdominal novocaine block suggested for dogs by L.G. Smirnov [10] and V.V. Reshetnyak [11] is defined by researches as "intraperitoneal" which is by puncturing of lateral abdominal wall. However, authors themselves acknowledge anatomical multidirectionality of activity of the block. With its irregular influence, it covers all the organs of an abdominal cavity at once - from a diaphragm to an entry into a pelvis. Such a multipolar affect cannot compete with suggested intraoperational paraaortal block of nerves of an abdominal aortic plexus in domestic dogs in the focus of influence.

Development of a connective-tissue capsule around the main nervous elements of an abdominal aortal plexus [12], as well as their isolation with adipose tissue demands targeted delivery of anesthetic solution to nerves and presence of elastic fibers in loose fibrous connective tissue surrounding the plexus talks for its ability to sizable stretching without disturbance of structural integrity.

The suggested technique of intraoperational paraaortal block doesn't bear the risk of injectable trauma (accurate visual and palpatory control) and overdose (it requires a small dose of anesthetic which is 5-6 times less than in sagittal irrigation to achieve clinical effect due to targeted delivery of solution to the object of impact). Coverage of all elements of an abdominal aortal plexus with color liquid (in more or less degree in case of injection either only in cephalic or in only caudal direction) causes the highest possible effectiveness of the method.

\section{Conclusion}

Macro-microanatomical and histological studies have shown allowed for development the technique of paraaortal surgical access to an abdominal aortal neurons plexus in pet dogs. The foregoing morphological reasoning proves the possibility of applying the developed technique of surgical access for block of nerves of celiac, cranial and caudal mesenteric and intermesenteric plexuses and sources of their formation with consideration to anathomotopographical patterns of neurogenic component of plexuses as well as epineural tissues.

The suggested technique of paraaortal surgical access allows for injection of anesthetic solutions into the area of the main autonomous innervation sources of abdominal and pelvic organs, which makes the basis for conduction of clinical studies of intersurgical paraaortal block of abdominal aortal plexus in pet dogs.

The studies were performed using the equipment of the collective use center of the Omsk State Autonomous State Institution "Endoscopic Surgery".

\section{References}

1. N.S. Bailard, J. Ortiz, R.A. Flores, Additives to local anaesthetics for peripheral nerve blocks: evidence, limitations and recommendations, American J. of Health-System Pharmacy, 71(5), 373-385 (2014)

2. K.A. Lemke, Local and regional analgesia, Veterinary Clinics of North America: Small Animal Practice, 32, 747-763 (2000)

3. E.A. Chandler, D.J. Thompson, J.B. Sutton, C.J. Price, eds., Canine Medicine and Therapeutics, $3^{\text {rd }}$ edition (Blackwell Science Ltd., London, 1995) 876 p.

4. M.A. Gurney, E.A. Leece, Analgesia for pelvic limb surgery. A review of peripheral nerve blocks and the extradural technique, Veterinary Anaesthesia and Analgesia, 41, 445-458 (2014)

5. H. Shebits, V. Brass, Operational surgery of dogs and cats (Aquarium Publ., Moscow, 2001) 512 p.

6. H.G. Nimand, P.F. Nimand, Dogs' illnesses: the practical guidance for veterinarians, $8^{\text {th }}$ ed. (Aquarium Publ., Moscow, 2001) 816 p.

7. O.R. Skubko, G.A. Khonin, O.N. Shushakova, S.F. Meleshkov, L.F. Bodrova, Morphological substantiation and technique of lumbar surgical approach for ovarian and uterine extraorganic nerve block in domestic dogs and domestic cats, J. of Pharmaceutical Sciences and Research, 10(7), 1727-1730 (2018)

8. A.F. Sapozhnikov et al., Local anesthesia and methods of novocain therapy in animals: guidance manual (Lan, Saint-Petersburg, 2011) 176 p.

9. L.G. Smirnov et al., Reasoning of intraperitoneal injection of Novocain for the purpose of curation of dogs, in: Proc. of Kostroma State Agricultural Academy, 58, 20-25 (2000)

10. V.V. Rechetnyak, The methods of an abdominal injection of Novocain in $\operatorname{dogs}$, in: Topical Issues of the Science in the AIC: Abstracts of the Interuniversity Sci.-Pract. Conf. (2000) p. 145

11. V.V. Semechenko, S.A. Barashkova, V.N. Artem'ev, Histological technique: guidance manual (Omsk State Medical Academy Publ., Omsk, 2003) pp. 6162, 66-67, 69-70

12. S.I. Shvedov, D.K. Ovchinnikov, Morphology of nerves forming an abdominal aortal plexus in canids and hares, in: Achievements of comparative, age-related and species morphology in veterinary medical practice: Proc. dedicated to professor $Y u$. F. Yudichev's 80 th anniversary (Omsk, 2011) pp. 229-234 